\title{
Entanglement echo and dynamical entanglement transitions
}

\author{
Kim Pöyhönen $\odot$ and Teemu Ojanen $\odot$ \\ Computational Physics Laboratory, Physics Unit, Faculty of Engineering and Natural Sciences, \\ Tampere University, P.O. Box 692, FI-33014 Tampere, Finland \\ and Helsinki Institute of Physics P.O. Box 64, FI-00014, Finland
}

(Received 21 June 2021; accepted 21 October 2021; published 18 November 2021)

\begin{abstract}
We formulate dynamical phase transitions in subsystems embedded in larger quantum systems. Introducing the entanglement echo as an overlap of the initial and instantaneous entanglement ground states, we show its analytic structure after a quench provides natural definition of dynamical phase transitions in the subsystem. These transitions come in two varieties: the entanglement-type transitions and the bulk-type Loschmidt transitions. The entanglement-type transitions arise from periodic reorganization of quantum correlations between the subsystem and its environment, manifesting in instantaneous entanglement ground state degeneracies. Furthermore, the entanglement echo distinguishes the direction of the quench, resolves spatially distinct dynamical phase transitions for nonuniform quenches, and give rise to sharply defined transitions for mixed initial states. We propose an experimental probe to identify entanglement-type transitions through temporal changes in subsystem fluctuations.
\end{abstract}

DOI: 10.1103/PhysRevResearch.3.L042027

Introduction. The rapidly growing field of dynamical quantum phase transitions aims to uncover general principles in nonequilibrium many-body dynamics and explore the parallels between dynamics and critical phenomena [1-8]. While there is no direct relation between far-from-equilibrium dynamics and equilibrium phases of matter, recent efforts have revealed a wealth of connections between them [9]. Moreover, in the modern age of quantum simulation and synthetic designer systems, theoretical predictions are directly stimulating new experimental directions [10-17]. Unifying themes across various subfields, dynamical phase transitions have an extraordinarily wide appeal in current research.

In the present work, we develop the theory of dynamical phase transitions of subsystems of larger many-body systems following a sudden quench, schematically illustrated in Fig. 1(a). In the diagnostics of quantum correlations in many-body systems, the entanglement spectrum has become an invaluable tool $[18,19]$. Recently, it has also found applications in far-from-equilibrium systems [20-28]. Here we consider a bipartite system and introduce the entanglement echo $\mathcal{E}(t)=\left\langle\lambda_{0}(0) \mid \lambda_{0}(t)\right\rangle$ as an overlap of the initial and instantaneous ground states $\left|\lambda_{0}\right\rangle$ of the entanglement Hamiltonian of a subsystem. We show that a vanishing entanglement echo at time $t_{c}$ provides a natural definition of dynamical phase transitions in a subsystem. The entanglement echo contains essential information on quantum correlations that is not

Published by the American Physical Society under the terms of the Creative Commons Attribution 4.0 International license. Further distribution of this work must maintain attribution to the author(s) and the published article's title, journal citation, and DOI. captured by the much-studied Loschmidt echo [1] and signals novel observable properties.

By solving dynamical entanglement transitions in onedimensional (1D) and two-dimensional (2D) topological lattice models, we demonstrate substantial conceptual advances in the theory of dynamical phase transitions. Most importantly, (i) the zeros of the entanglement echo exhibit two types of dynamical criticality as depicted in Fig. 1(b), the usual Loschmidt-type bulk transitions and the entanglementtype transitions which indicate periodic redistribution of quantum correlations between the subsystems and have no closed system analogy, (ii) inhomogeneous systems or macroscopically nonuniform quenches give rise to distinct robust spatially resolved dynamical phase transitions as illustrated in Fig. 1(c), and (iii) the entanglement-type transition can be probed by monitoring the temporal behavior of subsystem fluctuations [Fig. 1(d)] which also gives rise to oscillating entanglement entropy. In addition, the entanglement echo provides a natural framework to study entanglement transitions of systems in mixed states and nonunitary evolution.

Entanglement echo. To formulate a subsystem's dynamical phase transitions, we consider a bipartioning of a timeevolving system into two subsystems $A$ and $B$. The properties of the subsystem $A$ is encoded in the reduced density matrix $\rho_{A}(t)=\operatorname{Tr}_{B} \rho(t)=\sum_{i} \lambda_{i}(t)\left|\lambda_{i}(t)\right\rangle\left\langle\lambda_{i}(t)\right|$, obtained by tracing out the degrees of freedom corresponding to the subsystem $B$ from the full density matrix $\rho$ (representing a pure or mixed state). The reduced density matrix can be parametrized by the entanglement Hamiltonian $H_{E}$ defined by $\rho_{A}=\frac{e^{-H_{E}}}{Z}$, where $Z$ ensures the normalization $\operatorname{Tr}_{A} \rho_{A}=1$. The dominant contribution to $\rho_{A}$ comes from the state $\left|\lambda_{0}\right\rangle$ with the largest eigenvalue $\lambda_{0}$, corresponding to the ground state of the entanglement Hamiltonian. The entanglement ground state calculated for a many-body ground state typically 
(a)

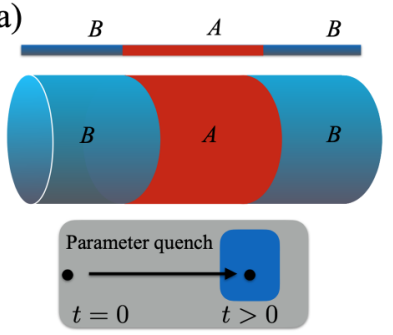

(c)

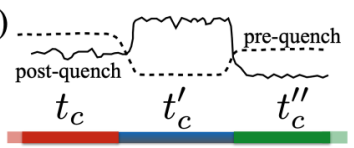

(b)

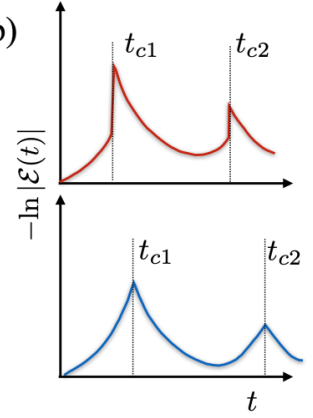

(d)

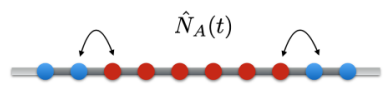

FIG. 1. (a) Dynamics of subsystem $A$ embedded in a larger system (seen here for 1D and 2D geometries) display singular features after a sudden quench through a critical point. (b) Entanglement echo $\mathcal{E}(t)$ resolves two types of dynamical criticality: an entanglement-type transition (top) and a bulk-type transition (bottom). (c) Entanglement echo can spatially resolve several dynamical transitions for a single quench configuration (solid and dashed lines). (d) Entanglement-type transitions can be probed by monitoring temporal change in the subsystem observables such as number of particles.

encodes universal information about the phase, such as topology and low-lying excitations. The significance of the entanglement ground state points to its potential importance also in far-from-equilibrium systems. Thus, we define the entanglement echo by

$$
\mathcal{E}(t)=\left\langle\lambda_{0}(0) \mid \lambda_{0}(t)\right\rangle,
$$

which measures the overlap between the initial and instantaneous entanglement ground states during temporal evolution. If the entanglement ground state is degenerate in the thermodynamic limit, the echo can be defined as the overlap with the degenerate subspaces.

As depicted in Fig. 1(a), we consider quench protocols where at $t=0$ the state of the whole system is prepared to a known initial state, such as the ground state or finitetemperature state of a prequench Hamiltonian. Then, the Hamiltonian of the system is instantaneously modified to the postquench form. Analogous to the Loschmidt echo $\mathcal{L}(t)=$ $\langle\Psi(0) \mid \Psi(t)\rangle$, the vanishing of which defines dynamical phase transitions for the full system, we define dynamical phase transitions for a subsystem in terms of the entanglement echo. We regard the subsystem $A$ as undergoing a dynamical phase transition at time $t_{c}$ if the entanglement echo vanishes $\mathcal{E}\left(t_{c}\right)=$ 0 . It is convenient to define the entanglement rate function $\Gamma(t)=-\ln |\mathcal{E}(t)|^{2} / \Omega_{A}$, where $\Omega_{A}$ is the characteristic size of the subsystem $A$. Dynamical phase transitions are clearly seen in the nonanalytic behavior of $\Gamma(t)$.

Here we describe two methods of calculating the entanglement echo. In the case of pure initial states and unitary evolution, the state of the system can be expanded $|\Psi(t)\rangle=$ $\sum_{\mu \nu} M_{\mu \nu}(t)\left|\psi_{\mu}^{A}\right\rangle\left|\psi_{\nu}^{B}\right\rangle$, where $\left|\psi_{\mu}^{A}\right\rangle,\left|\psi_{\nu}^{B}\right\rangle$ form a complete basis of each subsystem. The singular-value decomposition of matrix $M_{\mu \nu}(t)$ leads to the Schmidt decomposition of a

state as

$$
|\Psi(t)\rangle=\sum_{i} \lambda_{i}(t)^{1 / 2}\left|\lambda_{i}(t)\right\rangle\left|\lambda_{i}^{B}(t)\right\rangle,
$$

where the sum contains at most $\min (\operatorname{dim} A, \operatorname{dim} B)$ terms [29]. The entanglement ground state at time $t$ can be readily read off from (2), allowing a direct evaluation of the entanglement echo (1).

For noninteracting fermions, the evaluation of the entanglement echo simplifies. Pioneered by Peschel [30,31], the entanglement spectrum for free fermions in a Gaussian state can be obtained from the correlation matrix $\mathcal{C}_{l m}^{\sigma \sigma^{\prime}}=\left\langle\hat{c}_{l \sigma}^{\dagger} \hat{c}_{m \sigma^{\prime}}\right\rangle$, where fermion operators $\hat{c}_{m \sigma}$ annihilate particles with spin $\sigma$ and $l, m$ label positions in the subsystem $A$. The eigenstates $\left|\xi_{i}\right\rangle$ and eigenvalues $\xi_{i} \in[0,1]$ of the correlation matrix can be regarded as the eigenstates and occupation probabilities of a single-particle entanglement Hamiltonian. To evaluate the entanglement echo, we first need to compute the dynamical correlation matrix $\mathcal{C}_{l m}^{\sigma \sigma^{\prime}}(t)=\left\langle\hat{c}_{l \sigma}^{\dagger}(t) \hat{c}_{m \sigma^{\prime}}(t)\right\rangle$ and diagonalize it. The value $\xi=\frac{1}{2}$ marks the Fermi level of the entanglement Hamiltonian, so the entanglement ground state $\left|\lambda_{0}(t)\right\rangle$ is a Slater determinant constructed from the states satisfying $\frac{1}{2} \leqslant \xi(t) \leqslant 1$. In the second-quantized notation, it can be expressed as $\left|\lambda_{0}(t)\right\rangle=\prod_{\xi_{i}(t) \geqslant \frac{1}{2}} \hat{c}_{\xi_{i}(t)}^{\dagger}|0\rangle$. Then, the entanglement echo $\mathcal{E}(t)=\left\langle\lambda_{0}(0) \mid \lambda_{0}(t)\right\rangle$ becomes

$$
\mathcal{E}(t)=\operatorname{det}\left\langle\xi_{i}(0) \mid \xi_{j}(t)\right\rangle,
$$

where the single-particle states satisfy $\xi_{i}(0), \xi_{j}(t) \geqslant \frac{1}{2}$. This formula applies to zero- as well as to finite-temperature prequench states.

Dynamical entanglement transitions in $1 D$ and $2 D$. Now we demonstrate dynamical entanglement phase transitions in solvable two-band Fermi systems. Our analysis applies to arbitrary spatial dimensions but we focus on 1D and 2D topological lattice models. We consider pre- and postquench Hamiltonians of the form

$$
H^{i / f}=\sum_{l, m} \hat{\boldsymbol{c}}_{l}^{\dagger}\left[\boldsymbol{d}_{l m}^{i / f} \cdot \boldsymbol{\sigma}\right] \hat{\boldsymbol{c}}_{m},
$$

where $\sigma=\left(I, \sigma_{x}, \sigma_{y}, \sigma_{z}\right)$ is a vector of Pauli matrices and the set of matrices $\boldsymbol{d}^{i / f}=\left(d_{0}^{i / f}, d_{x}^{i / f}, d_{y}^{i / f}, d_{z}^{i / f}\right)$ determine the specific form of pre- $\left(H^{i}\right)$ and post- $\left(H^{f}\right)$ quench Hamiltonians. The spinor operator $\hat{\boldsymbol{c}}_{l}^{\dagger}=\left(\hat{c}_{l \uparrow}^{\dagger}, \hat{c}_{l \downarrow}^{\dagger}\right)$ creates fermions at site $l$. For translationally invariant systems and quenches, matrices $\boldsymbol{d}^{i / f}$ become diagonal in an $n$-dimensional quasimomentum space. With a minor modification, which extends spinors $\hat{\boldsymbol{c}}$ to the Nambu space, model (4) also describes quenches in topological superconductors and solvable spin chains $[1,32,33]$ and spin liquids [34]. In Sec. I of the Supplemental Material (SM) [35], we have derived the expression for the dynamic correlation matrix for the model (4) for spatially dependent parameters and quench protocols in zero and finitetemperature initial states.

We first consider a 1D topological insulator defined by $\boldsymbol{d}(k)=(0, \sin k, 0, m-\cos k)$. This model belongs to the Altland-Zirnbauer class BDI [36,37] and exhibits a nontrivial phase for $|m|<1$ and trivial phase for $|m|>1$. In Figs. 2(a) and 2(b) we have illustrated the dynamical phase transitions when the system is quenched through the critical 

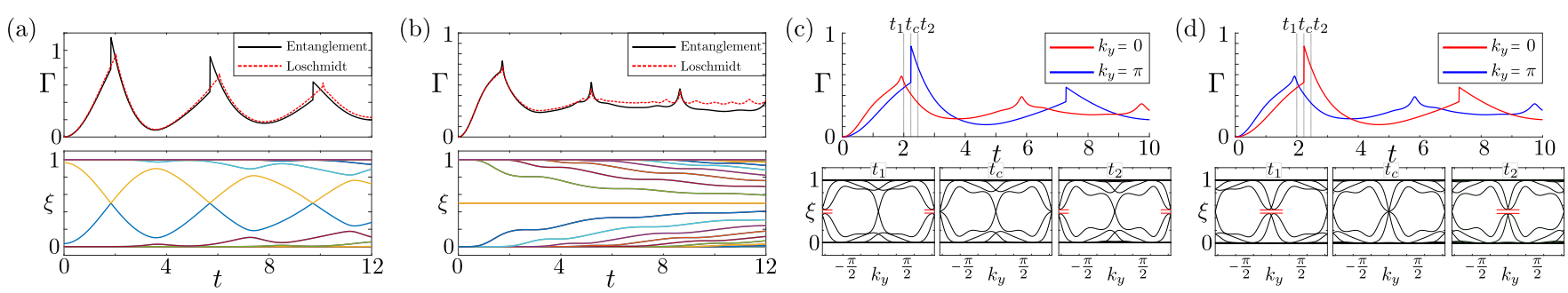

FIG. 2. (a) Entanglement echo rate of a 1D system (top) for quench $m=1.5 \rightarrow m=0.3$ and the system size $L=100$ (total), $L_{A}=30$ (subsystem). The jump singularities, which arise from the crossing of the entanglement spectrum (bottom), do not coincide with the cusps singularities of the Loschmidt echo of the full system. (b) Same as (a) but for the opposite quench $m=0.3 \rightarrow m=1.5$. The two echoes agree (apart from finite size effects which vanish in the thermodynamic limit). (c) Momentum-resolved entanglement echo rate (top) for Chern insulator quench $m=0.5 \rightarrow m=-0.5$ and the system size $L=100$ (total), $L_{A}=30$ (subsystem). The jump singularities correspond to $k_{y}$ values for which the entanglement spectrum (bottom) exhibits temporal gap closings at $t_{c}$. (d) Same as (c) but for the opposite quench $m=-0.5 \rightarrow m=0.5$.

point $m=1$. The entanglement echo distinguishes whether the quench is performed from the trivial to the topological phase or vice versa. In the former case, which we dub as an entanglement-type transition, the entanglement echo displays periodic jump discontinuities as shown in Fig. 2(a). The entanglement spectrum reveals that the jumps arise from stroboscopic level crossing signaling an instantaneous entanglement ground state degeneracy. In the latter case [Fig. 2(b)], which we call a bulk-type transition, the entanglement echo exhibits cusps and agrees with the Loschmidt rate function. The main difference between the two types of transition is that the entanglement ground state degeneracy is oscillating and present only at critical times for the entanglement-type transitions. In contrast, the degeneracy remains static for bulktype transitions for timescales comparable to the critical time, which is manifested in Fig. 2(b) (bottom) by the static states pinned to the midgap. The fact that the midgap states appear frozen in the topological-to-trivial quench follows from the fact that they represent edge states that are localized to the different ends of the system. The degeneracy of the midgap states present in the topologically nontrivial initial state can be lifted only after the information of the quench has had time to propagate through the system [25]. The propagation velocity obeys a Lieb-Robinson-type bound, so the timescale for which the midgap states remain frozen diverges when the subsystem becomes thermodynamically large. The bulkand entanglement-type transitions are mutually exclusive in the sense that the same subsystem can exhibit either one (or neither) but not both simultaneously. The oscillating entanglement ground state degeneracy modifies a bulk-type transition to an entanglement-type transition.

As shown in Sec. V of the SM [35], the stroboscopic entanglement ground state degeneracies persist also to finitetemperature initial states. In contrast to the Loschmidt echo, which does not offer a straightforward generalization with sharply defined transitions at finite temperatures [38-42], the entanglement-type transitions remain well defined. The interferometric phase approach proposed in $[38,40]$ gives rise to nonanalytic behavior at finite temperatures but is not sensitive to entanglement properties captured by the entanglement echo. While the entanglement echo for the bulk-type transitions reduce to the Loschmidt echo of the total system, as seen in Fig. 2(a) and discussed in Sec. III of the SM [35], the analytic structure and the critical times of entanglement-type transitions do not coincide with the Loschmidt transitions. The entanglement echo quantifies a temporal reorganization of quantum correlations between the two subsystems and captures essential information not contained in the Loschmidt echo. When the subsystem size approaches the full system size, naturally, the entanglement-type transition becomes a bulk-type transition. Assuming periodic boundary conditions of the full system, the crossover from a jump to cusp singularity happens approximately when the length of the segment that does not belong to the subsystem is comparable to the characteristic penetration depth of the edge modes of the postquench Hamiltonian.

Two-dimensional systems exhibit similar bulk- and entanglement-type transitions as 1D systems. By considering the geometry shown in Fig. 1(a), the subsystem $A$ can be chosen as a segment in the $x$ direction so that the perpendicular momentum $k_{y}$ remains a good quantum number. The reduced density matrix decouples to blocks labeled by $k_{y}$, and the entanglement echo can be decomposed from the echoes of each block as $\mathcal{E}(t)=\prod_{k_{y}} \mathcal{E}\left(k_{y}, t\right)$. The $k_{y^{-}}$ resolved partial echoes can be obtained by diagonalizing the momentum-resolved correlation matrix derived in Sec. I of the SM [35]. After obtaining the eigenfunctions $\left|\xi_{i}\left(k_{y}, t\right)\right\rangle$, the partial echoes can be calculated by applying Eq. (3). In fact, quench dynamics are conveniently analyzed in terms of partial echoes $\mathcal{E}\left(k_{y}, t\right)$. Here we consider Chern insulators defined by $\boldsymbol{d}(k)=\left(0, \sin k_{x}, \sin k_{y}, m-\cos k_{x}-\cos k_{y}\right)$, which exhibits three distinct topological phases with Chern numbers $C=-1$ (when $-2<m<0$ ), $C=1(0<m<2$ ), and $C=0$ (when $|m|>2)$. As in the 1D case, when the system is quenched through a critical point, the entanglement echo shows nonanalytic behavior which depends on the direction of the quench. In addition, the entanglement echo rate function may exhibit either a cusp or jump singularity depending on $k_{y}$. This is illustrated in Figs. 2(c) and 2(d) for transitions between $C= \pm 1$ phases. At times when the rate function shows a jump singularity for specific $k_{y}$ values, the instantaneous entanglement spectrum exhibits temporal gap closing for the corresponding $k_{y}$. Also, the momentum for which the gap closing takes place changes when the direction of the quench is inverted. Thus, the entanglement echo in both $1 \mathrm{D}$ and 2D systems reveals two distinct dynamical phase transitions and, in contrast to 


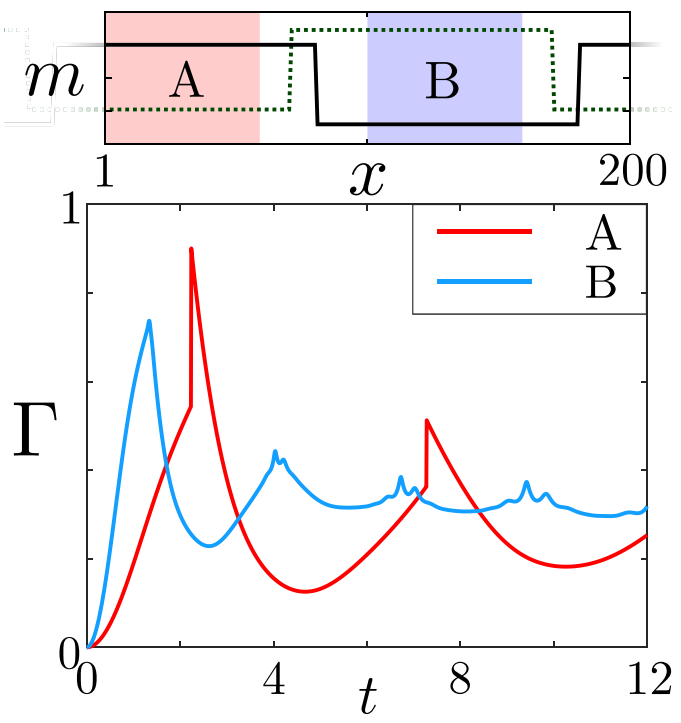

FIG. 3. Top: Spatially varying prequench (solid) and postquench (dashed) configurations for the 1D model with periodic boundary conditions. The initial configuration consists of regions with $\mu_{1}=$ 1.5 and $\mu_{2}=0.3$, while the postquench parameters have $\mu_{3}=0.5$ and $\mu_{4}=1.7$. Bottom: Resulting distinct transitions displayed in subsystems $A$ (red) and $B$ (blue).

the Loschmidt echo, makes a qualitative distinction in which direction the critical point is crossed.

Spatially varying quenches. The Loschmidt echo characterizes dynamics of the system as a whole and, as such, is incapable of providing spatially resolved information. However, the entanglement echo reveals novel dynamical criticality in macroscopically inhomogeneous systems or spatially varying quenches. In fact, a single quench can give rise to several spatially resolved dynamical phase transitions characterized by different timescales. In Fig. 3 we have illustrated a quench in the 1D system, where the pre- and postquench configurations vary in space. The subsystem $A$ experiences a quench from a trivial to topological phase while subsystem $B$ experiences the opposite quench. The entanglement echo reveals that, indeed, the different parts of the system exhibit distinct sharply defined dynamical phase transitions. Not only are their critical times different, but the nonanalytic structure shows that the transition in $A$ is of entanglement type and the transition in $B$ is of bulk type. Since it takes a finite time for information to propagate through the system [25], the short-time behavior giving rise to early dynamical phase transitions is sensitive only to local quench properties. Thus, a system which exhibits several distinct equilibrium critical points can display multiple spatially resolved dynamical phase transitions in a single quench. As mentioned above in the case of spatially homogeneous systems, each subsystem can be well defined as either bulk or entanglement type as long as the topological phase of the subsystem is unambiguous, as in Fig. 3.

Observable consequences. The entanglement-type transitions arise from instantaneous degeneracies of the entanglement ground state which persist to finite-temperature initial states. It is natural to wonder what the observable
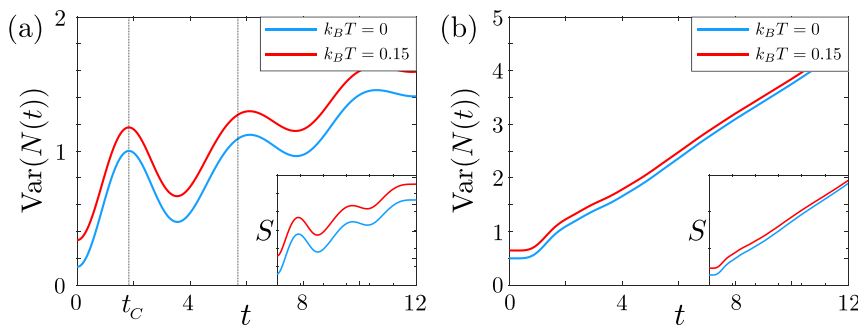

FIG. 4. Subsystem particle number variance and entanglement entropy (inset) after a quench. (a) corresponds to the entanglementtype transition in Figs. 1(a) and 1(b) to the bulk-type transition in Fig. 1(b).

consequences of this are, especially in contrast to the Loschmidt-type criticality. Far from equilibrium, the states in the entanglement spectrum are not in simple correspondence with the physical edge modes, thus preventing the most direct experimental probes. Here we devise a method to probe and distinguish the entanglement-type transitions by monitoring the temporal changes in subsystem fluctuations. The stroboscopic degeneracy of two entanglement ground states is expected to lead to enhanced fluctuations for observables which have different expectation values in the two states. Indeed, we demonstrate this by considering the number of particles in the subsystem $A$ in a setup depicted in Fig. 1(d). The particle number operator is $\hat{N}_{A}=\sum_{i \in A, \alpha=\uparrow, \downarrow} \hat{c}_{i \alpha}^{\dagger} \hat{c}_{i \alpha}$, where the summation is over the lattice sites in $A$ and spin. As shown in Sec. IV of the SM [35], the time-dependent variance of the particle number is given by

$$
\operatorname{Var} N_{A}(t)=\sum_{i}\left[\xi_{i}(t)-\xi_{i}^{2}(t)\right]
$$

where $\xi_{i}(t)$ are the eigenvalues of the correlation matrix. In a translation-invariant system, the first term is a constant fixed by the average density; however, the second term should reflect the pronounced oscillations of midgap states characterizing the entanglement-type transitions shown in Fig. 2(a). As seen in Fig. 4(a), the particle number variance indeed oscillates with periodicity of the critical times. In addition to oscillations, it shows a linear trend due to mixing of the two subsystems. The onset time of the linear growth depends on the depth of a quench while the oscillation period reflects the periodicity of critical times. The pronounced oscillations, which are visible even for small subsystems down to approximately ten sites, persist to finite-temperature initial states and provide an experimental signal that distinguishes entanglement-type transitions from Loschmidt transitions seen in Fig. 4(b) and trivial quenches shown in Sec. V in the SM. Moreover, as illustrated in Fig. 4, particle number fluctuations essentially reflect the behavior of the von Neumann entropy $S(t)=-\sum_{i}\left[\xi_{i} \ln \xi_{i}+\left(1-\xi_{i}\right) \ln \left(1-\xi_{i}\right)\right]$ [43]. The difference in entropy oscillations [44] depending on the direction of the quench is naturally explained by the existence of the two types of subsystem transitions discussed in our work. Since the above discussed mechanism of the subsystem fluctuations follows from the oscillating entanglement ground state degeneracy, it applies to generic observables and entanglement-type dynamical phase transitions. In particular, 
the particle number oscillations in the entanglement-type transitions discussed above are robust in the presence of moderate interactions that do not destroy topological states.

Conclusion and outlook. In this work we formulated dynamical phase transitions for a subsystem of a many-body system by introducing the entanglement echo. The entanglement echo provides an appropriate generalization of the Loschmidt echo, giving rise to several conceptual advances as well as new observable predictions discussed in our work. In the present work, we studied dynamical criticality resulting from a zero- and finite-temperature initial state undergoing unitary time evolution after a quench. Since the entanglement echo is formulated in terms of the reduced density matrix, it can be straightforwardly employed to study mixed states and nonunitary time evolution. In the future, it will be interesting to study subsystem dynamics in systems subjected to measurements, the effects of measurements on quench dynamics [45], and possible measurement-induced dynamical entanglement phase transitions [46-48].

Acknowledgments. The authors acknowledge support from the Academy of Finland project 331094.
[1] M. Heyl, A. Polkovnikov, and S. Kehrein, Dynamical Quantum Phase Transitions in the Transverse-Field Ising Model, Phys. Rev. Lett. 110, 135704 (2013).

[2] C. Karrasch and D. Schuricht, Dynamical phase transitions after quenches in nonintegrable models, Phys. Rev. B 87, 195104 (2013).

[3] F. Andraschko and J. Sirker, Dynamical quantum phase transitions and the Loschmidt echo: A transfer matrix approach, Phys. Rev. B 89, 125120 (2014).

[4] S. Vajna and B. Dóra, Disentangling dynamical phase transitions from equilibrium phase transitions, Phys. Rev. B 89, 161105(R) (2014).

[5] S. Vajna and B. Dóra, Topological classification of dynamical phase transitions, Phys. Rev. B 91, 155127 (2015).

[6] J. C. Budich and M. Heyl, Dynamical topological order parameters far from equilibrium, Phys. Rev. B 93, 085416 (2016).

[7] A. A. Zvyagin, Dynamical quantum phase transitions (Review Article), J. Low Temp. Phys. 42, 971 (2016).

[8] J. C. Halimeh and V. Zauner-Stauber, Dynamical phase diagram of quantum spin chains with long-range interactions, Phys. Rev. B 96, 134427 (2017).

[9] M. Heyl, Dynamical quantum phase transitions: A review, Rep. Prog. Phys. 81, 054001 (2018).

[10] P. Jurcevic, H. Shen, P. Hauke, C. Maier, T. Brydges, C. Hempel, B. P. Lanyon, M. Heyl, R. Blatt, and C. F. Roos, Direct Observation of Dynamical Quantum Phase Transitions in an Interacting Many-Body System, Phys. Rev. Lett. 119, 080501 (2017).

[11] J. Zhang, G. Pagano, P. W. Hess, A. Kyprianidis, P. Becker, H. Kaplan, A. V. Gorshkov, Z.-X. Gong, and C. Monroe, Observation of a many-body dynamical phase transition with a 53-qubit quantum simulator, Nature (London) 551, 601 (2017).

[12] N. Flschner, D. Vogel, M. Tarnowski, B. S. Rem, D.-S. Lhmann, M. Heyl, J. C. Budich, L. Mathey, K. Sengstock, and C. Weitenberg, Observation of dynamical vortices after quenches in a system with topology, Nat. Phys. 14, 265 (2018).

[13] X.-Y. Guo, C. Yang, Y. Zeng, Y. Peng, H.-K. Li, H. Deng, Y.-R. Jin, S. Chen, D. Zheng, and H. Fan, Observation of a Dynamical Quantum Phase Transition by a Superconducting Qubit Simulation, Phys. Rev. Appl. 11, 044080 (2019).

[14] T. Tian, Y. Ke, L. Zhang, S. Lin, Z. Shi, P. Huang, C. Lee, and J. $\mathrm{Du}$, Observation of dynamical phase transitions in a topological nanomechanical system, Phys. Rev. B 100, 024310 (2019).

[15] K. Wang, X. Qiu, L. Xiao, X. Zhan, Z. Bian, W. Yi, and P. Xue, Simulating Dynamic Quantum Phase Transitions in Photonic Quantum Walks, Phys. Rev. Lett. 122, 020501 (2019).

[16] T. Tian, H.-X. Yang, L.-Y. Qiu, H.-Y. Liang, Y.-B. Yang, Y. Xu, and L.-M. Duan, Observation of Dynamical Quantum Phase Transitions with Correspondence in an Excited State Phase Diagram, Phys. Rev. Lett. 124, 043001 (2020).

[17] K. Xu, Z.-H. Sun, W. Liu, Y.-R. Zhang, H. Li, H. Dong, W. Ren, P. Zhang, F. Nori, D. Zheng, H. Fan, and H. Wang, Probing dynamical phase transitions with a superconducting quantum simulator, Sci. Adv. 6, eaba4935 (2020).

[18] H. Li and F. D. M. Haldane, Entanglement Spectrum as a Generalization of Entanglement Entropy: Identification of Topological Order in Non-Abelian Fractional Quantum Hall Effect States, Phys. Rev. Lett. 101, 010504 (2008).

[19] L. Fidkowski, Entanglement Spectrum of Topological Insulators and Superconductors, Phys. Rev. Lett. 104, 130502 (2010).

[20] G. Torlai, L. Tagliacozzo, and G. D. Chiara, Dynamics of the entanglement spectrum in spin chains, J. Stat. Mech.: Theory Exp. (2014) P06001.

[21] E. Canovi, E. Ercolessi, P. Naldesi, L. Taddia, and D. Vodola, Dynamics of entanglement entropy and entanglement spectrum crossing a quantum phase transition, Phys. Rev. B 89, 104303 (2014).

[22] R. Jafari, H. Johannesson, A. Langari, and M. A. MartinDelgado, Quench dynamics and zero-energy modes: The case of the Creutz model, Phys. Rev. B 99, 054302 (2019).

[23] S. De Nicola, A. A. Michailidis, and M. Serbyn, Entanglement View of Dynamical Quantum Phase Transitions, Phys. Rev. Lett. 126, 040602 (2021).

[24] Z. Gong and M. Ueda, Topological Entanglement-Spectrum Crossing in Quench Dynamics, Phys. Rev. Lett. 121, 250601 (2018).

[25] Z. Gong, N. Kura, M. Sato, and M. Ueda, Lieb-Robinson bounds on entanglement gaps from symmetry-protected topology, arXiv:1904.12464.

[26] S. Lu and J. Yu, Stability of entanglement-spectrum crossing in quench dynamics of one-dimensional gapped free-fermion systems, Phys. Rev. A 99, 033621 (2019).

[27] L. Pastori, S. Barbarino, and J. C. Budich, Signatures of topology in quantum quench dynamics and their interrelation, Phys. Rev. Research 2, 033259 (2020).

[28] J. Surace, L. Tagliacozzo, and E. Tonni, Operator content of entanglement spectra in the transverse field Ising chain after global quenches, Phys. Rev. B 101, 241107(R) (2020). 
[29] A. Ekert and P. L. Knight, Entangled quantum systems and the Schmidt decomposition, Am. J. Phys. 63, 415 (1995).

[30] I. Peschel, Calculation of reduced density matrices from correlation functions, J. Phys. A: Math. Gen. 36, L205 (2003).

[31] I. Peschel and V. Eisler, Reduced density matrices and entanglement entropy in free lattice models, J. Phys. A: Math. Theor. 42, 504003 (2009).

[32] K. Najafi, M. A. Rajabpour, and J. Viti, Return amplitude after a quantum quench in the XY chain, J. Stat. Mech. (2019) 083102.

[33] S. Zamani, R. Jafari, and A. Langari, Floquet dynamical quantum phase transition in the extended xy model: Nonadiabatic to adiabatic topological transition, Phys. Rev. B 102, 144306 (2020).

[34] M. Schmitt and S. Kehrein, Dynamical quantum phase transitions in the Kitaev honeycomb model, Phys. Rev. B 92, 075114 (2015).

[35] See Supplemental Material at http://link.aps.org/supplemental/ 10.1103/PhysRevResearch.3.L042027 for technical derivations and complementary results.

[36] A. Altland and M. R. Zirnbauer, Nonstandard symmetry classes in mesoscopic normal-superconducting hybrid structures, Phys. Rev. B 55, 1142 (1997).

[37] A. P. Schnyder, S. Ryu, A. Furusaki, and A. W. W. Ludwig, Classification of topological insulators and superconductors in three spatial dimensions, Phys. Rev. B 78, 195125 (2008).

[38] U. Bhattacharya, S. Bandyopadhyay, and A. Dutta, Mixed state dynamical quantum phase transitions, Phys. Rev. B 96, 180303(R) (2017).

[39] U. Bhattacharya and A. Dutta, Emergent topology and dynamical quantum phase transitions in two-dimensional closed quantum systems, Phys. Rev. B 96, 014302 (2017).

[40] M. Heyl and J. C. Budich, Dynamical topological quantum phase transitions for mixed states, Phys. Rev. B 96, 180304(R) (2017).

[41] N. Sedlmayr, M. Fleischhauer, and J. Sirker, Fate of dynamical phase transitions at finite temperatures and in open systems, Phys. Rev. B 97, 045147 (2018).

[42] N. O. Abeling and S. Kehrein, Quantum quench dynamics in the transverse field Ising model at nonzero temperatures, Phys. Rev. B 93, 104302 (2016).

[43] Y. F. Zhang, L. Sheng, R. Shen, R. Wang, and D. Y. Xing, Entanglement, subsystem particle numbers and topology in free fermion systems, J. Phys.: Condens. Matter 26, 105502 (2014).

[44] N. Sedlmayr, P. Jaeger, M. Maiti, and J. Sirker, Bulkboundary correspondence for dynamical phase transitions in one-dimensional topological insulators and superconductors, Phys. Rev. B 97, 064304 (2018).

[45] W.-T. Kuo, D. Arovas, S. Vishveshwara, and Y.-Z. You, Decoherent quench dynamics across quantum phase transitions, SciPost Phys. 11, 084 (2021).

[46] Y. Li, X. Chen, and M. P. A. Fisher, Quantum Zeno effect and the many-body entanglement transition, Phys. Rev. B 98, 205136 (2018).

[47] A. Chan, R. M. Nandkishore, M. Pretko, and G. Smith, Unitaryprojective entanglement dynamics, Phys. Rev. B 99, 224307 (2019).

[48] B. Skinner, J. Ruhman, and A. Nahum, Measurement-Induced Phase Transitions in the Dynamics of Entanglement, Phys. Rev. X 9, 031009 (2019). 